\title{
Nível de dano econômico de Sitophilus zeamais (M.) em trigo armazenado ${ }^{1}$
}

\author{
Anderson K. Santos ${ }^{2}$, Lêda R. D'Antonino Faroni ${ }^{3}$, Raul N. C. Guedes ${ }^{4}$, \\ Jamilton P. dos Santos ${ }^{5}$ \& Adriano F. Rozazdo ${ }^{6}$
}

\begin{abstract}
1 Parte da Dissertação de Mestrado do primeiro autor, apresentada à UFV.
2 Moinhos Vera Cruz S.A., Av. Ângelo Teixeira da Costa 1077, Distrito Industrial de Carreira Comprida, CEP 33045-170, Santa Luzia, MG. E-mail: aksantos@gold.com.br (Foto)

3 DEA/UFV. CEP 36571-000, Viçosa, MG. E-mail: Ifaroni@mail.ufv.br

4 DBA/UFV. CEP 36571-000, Viçosa, MG. E-mail: guedes@mail.ufv.br

${ }^{5}$ EMBRAPA Milho e Sorgo. Rod. MG 424, km 65, CEP 35701-970, Sete Lagoas, MG. E-mail: jamilton@cnpms.embrapa.br

${ }^{6}$ Graduando DEA/UFV. Viçosa, MG. E-mail: eg34922@correio.ufv.br
\end{abstract}

Protocolo $114-24 / 7 / 2001$

\begin{abstract}
Resumo: Calcular o nível de dano econômico (NDE) para o principal inseto-praga Sitophilus zeamais (Motschulsky, 1895) do trigo armazenado, foi o objetivo deste trabalho. Para o cálculo do NDE relacionaram-se o grau de infestação e as perdas qualitativa e quantitativa dos grãos, depois de três meses de armazenamento. Utilizaram-se potes de vidro transparente de $2,5 \mathrm{~L}$, com 1,5 kg de grãos de trigo tipo durum, da safra de setembro/97, com teor de umidade de $12,5 \%$. Os potes foram infestados com densidades populacionais de $0,2,10,20,30,50,100,200$ e 300 insetos da espécie $S$. zeamais, em três repetições, e então armazenados por um período de 90 d, em câmaras tipo BOD, com temperatura de $27{ }^{\circ} \mathrm{C}$ e $65 \pm 5 \%$ de umidade relativa. Logo depois do período de armazenamento tomava-se a temperatura da massa de grãos e, em seguida, contava-se o número de insetos adultos vivos e mortos. Os grãos foram analisados quanto à perda de peso e à perda de qualidade (peso hectolítrico e teor de umidade). Tanto a massa final quanto o peso hectolítrico ( $\mathrm{PH}$ ) para cada nível de infestação, foram corrigidos para o teor de umidade inicial dos grãos. Utilizando-se os dados do valor de mercado do grão de trigo importado e nacional, do custo de controle para fumigação e do valor obtido pela inclinação das curvas de regressão entre perda de peso $(\mathrm{g})$ e $\mathrm{PH}\left(\mathrm{g} 250 \mathrm{~mL}^{-1}\right)$ em relação à infestação final de $S$. zeamais, calculou-se o NDE. Com base na redução do peso da massa de grãos, no peso hectolítrico e no valor de mercado do trigo, encontrou-se um NDE de 0,18 inseto $\mathrm{kg}^{-1}$ de grão.
\end{abstract}

Palavras-chave: armazenamento de trigo; perda de qualidade; infestação de insetos.

\section{Estimation of the level of economic loss for Sitophilus zeamais (M.) in stored wheat}

\begin{abstract}
The objective of this study was to evaluate the economic loss level (ELL) for the main insect-pest of stored wheat - Sitophilus zeamais (Motschulsky, 1895). In order to achieve this, the level of infestation was related to qualitative and quantitative losses after three months of storage. Glass containers $(2.5 \mathrm{~L})$ were filled with $1.5 \mathrm{~kg}$ wheat (type "durum"; harvested in Sept., 1997) with $12.5 \%$ moisture content. The wheat was infested with 0, 2, 10, 20, 30, 50, 100, 200, and 300 individuals of $S$. zeamais, in three replications. The glass containers containing infested wheat were stored for 90 days in BOD chambers at $27{ }^{\circ} \mathrm{C}$ and $65 \pm 5 \%$ relative humidity. After the storage period, the grain temperature was measured and the number of live and dead insects was counted. Grain weight loss and quality (moisture content and "bulk density") were analyzed. The final mass and the bulk density were corrected for the initial moisture content. The market value of national and imported wheat, the cost of fumigation as well as the regression coeficients between grain weight and quality losses, and insect infestation were used to estimate the ELL. Based on the grain weight loss, bulk density, and the market value of wheat, the ELL was 0.18 insects $\mathrm{kg}^{-1}$ of grain.
\end{abstract}

Key words: stored wheat; qualitative loss; infestation of insects

\section{INTRODUÇÃO}

Grãos de cereais e seus subprodutos estão sujeitos ao ataque de pragas, que causam perdas qualitativas e quantitativas
(Pedersen, 1992) reduzindo os valores nutritivos e comerciais do produto (Anderson et al., 1990; Reed et al. 1990). Segundo Sinha (1995) e Schöller et al. (1997) as perdas podem atingir até $30 \%$ em alguns casos, sendo $10 \%$ causados diretamente pelo 
ataque de pragas durante o armazenamento. De acordo com Hagstrum \& Flinn (1992) a maioria dessas pragas tem taxa de desenvolvimento capaz de multiplicar a população inicial em pelo menos 10 vezes por mês, sob condições ótimas.

Em todo o mundo, a forma mais utilizada para a proteção de grãos armazenados contra a infestação de pragas é o controle químico (White \& Leesch, 1996) por ser efetivo, de baixo custo e de fácil manejo (Harein \& Davis, 1992); no entanto, o crescente desenvolvimento de resistência dos insetos aos agentes químicos, a possibilidade de intoxicação dos operadores e a presença de resíduos nos alimentos, levaram à busca de alternativas para se alcançar o maior nível de proteção da saúde humana e que proporcionassem menor impacto ambiental (Brower et al., 1996). Em razão disso, unidades armazenadoras e processadoras de grãos têm procurado adotar o Manejo Integrado de Pragas (MIP) em que métodos higiênicos e sanitários, tecnológicos, físicos, químicos e biológicos, são combinados para se alcançar o maior nível de proteção da saúde humana, do ambiente e do produto (Hagstrum \& Flinn, 1996).

Um programa de manejo integrado de pragas, de acordo com Hagstrum \& Flinn (1996) envolve o monitoramento de insetos, análises de risco e de custo-benefício para a tomada de decisões, além do uso de múltiplas táticas de controle. Ainda segundo os mesmos autores, em programas de manejo integrado, o controle é economicamente eficiente se o custo de controle for menor que a redução no valor de mercado, causada pela praga. As análises de custo-benefício para o manejo integrado de pragas são baseadas em dois limites, segundo Hagstrum \& Flinn (1996): no Nível de Dano Econômico (NDE) e no Nível de Controle (NC).

Definido por Stern et al. (1959) como sendo a menor densidade populacional possível de causar dano econômico, o NDE apresenta dificuldades para ser determinado (Chant, 1966). Stern (1966) apresentou três métodos empíricos de se estabelecer esses níveis, sendo que cada um requeria estimativas visuais de perda. Um quarto método pode ser desenvolvido integrando custo, valor de mercado e dados de produtividade. Este método dedutivo para a determinação do NDE em laboratório permite a manipulação experimental dos fatores que influenciam tais níveis (Hagstrum \& Flinn, 1992). O nível de controle (NC) é a densidade do inseto na qual o controle deve ser aplicado para evitar que o dano ou a contaminação alcance o NDE. O NC permite adiantar a tomada de decisão de se aplicar medidas de controle e o tempo requerido para que a medida de controle reduza a densidade do inseto (Onstad, 1987).

O NDE pode ser calculado utilizando-se tanto a perda da qualidade quanto a perda de peso dos grãos. Na perda de qualidade leva-se em consideração as características do produto envolvidas no valor comercial, como o peso hectolítrico $(\mathrm{PH})$ para trigo nacional e proteína para trigo argentino (Brasil, 1998). A perda de peso pode ser avaliada pelo grau de infestação por insetos. Os insetos, durante o seu desenvolvimento, alimentam-se principalmente do endosperma dos grãos e, conseqüentemente, o rendimento em farinha, obtida desses grãos, será menor (Olkowski, 1974; Zungoli \& Robinson, 1984; Raupp et al., 1987; Hagstrum \& Flinn, 1992; Hagstrum \& Flinn, 1996).
As indústrias alimentícias têm desenvolvido um programa de controle e análise de pontos críticos (HACCP ou APPCC) em sua linha de produção, para assegurar a qualidade dos seus produtos (Mills \& Pedersen, 1990) cuja finalidade e parâmetros se assemelham ao NDE e LE do MIP. A diferença entre os dois está na ênfase de identificação dos pontos críticos do sistema, no qual o HACCP enfatiza o nível crítico e o desenvolvimento de programas que evitem o início e a proliferação de riscos, enquanto o MIP enfatiza o uso da análise de custo-benefício para determinar se o controle é um custo efetivo. O HACCP engloba o MIP no processo, pois as pragas, além do prejuízo, podem causar risco à saúde humana, de forma direta (infecção) ou indireta (resíduos de inseticidas).

Em razão do exposto, o objetivo desse estudo foi calcular o NDE para o principal inseto-praga, Sitophilus zeamais (Motschulsky, 1895) do trigo armazenado no Brasil. Para o cálculo do NDE relacionaram-se o grau de infestação e as perdas qualitativa e quantitativa dos grãos, depois de três meses de armazenamento.

\section{MATERIAL E MÉTODOS}

O experimento foi realizado no Laboratório de Processamento de Grãos do Departamento de Engenharia Agrícola da Universidade Federal de Viçosa, Viçosa, MG. Em potes de vidro transparente de 2,5 L, foi colocado $1,5 \mathrm{~kg}$ de grãos de trigo importado Argentino da safra de 1997 com teor de umidade de $12,5 \%$. Esta quantidade de grãos foi suficiente para a determinação do peso hectolítrico e para o teor de umidade.

Os potes foram infestados com densidades populacionais de $0,2,10,20,30,50,100,150,200,250$ e 300 insetos da espécie $S$. zeamais, em três repetições, adultos jovens (recémemergidos) porém não sexados, coletados de uma população mantida sob as condições de $27^{\circ} \mathrm{C}$ de temperatura e $65 \pm 5 \%$ de umidade relativa, iguais às do experimento.

Os potes infestados foram então armazenados por um período de 90 dias, de junho a agosto de 1998, que se refere à média de meses cujo produto permanece em armazenamento em indústrias moageiras, podendo, no entanto, ser armazenados por períodos bem mais prolongados; depois foram armazenados em câmaras tipo BOD, com controle de temperatura. A umidade relativa (UR) foi estabelecida em $65 \pm$ $5 \%$, de tal forma a manter a umidade de equilíbrio dos grãos. A temperatura de $27^{\circ} \mathrm{C}$ e $70 \%$ de UR contempla as condições ótimas de desenvolvimento do S. zeamais (Rees, 1996) para grãos com 13\% de umidade (Okelana \& Osuji, 1985).

Depois do período de armazenamento, a população de insetos e a massa de grãos foram avaliadas. Inicialmente, tomava-se a temperatura da massa de grãos, com um termômetro digital; em seguida, contaram-se os insetos adultos vivos e mortos, utilizando-se a relação de 1220 insetos para cada $5 \mathrm{~mL}$ de volume de insetos inseridos em uma proveta de $50 \mathrm{~mL}$ (esta relação foi obtida depois de vários ensaios em laboratório). Esta avaliação é fundamental para a determinação dos Níveis de Danos. Para isso, a massa de 1,5 kg de trigo de cada pote foi peneirada em peneira de crivos oblongos, de 1,41 mm, para separar os insetos dos grãos. O único parâmetro populacional 
adotado foi o número de insetos adultos, não sendo acompanhados os demais estágios, como ovos, larvas e pupas. Depois, os grãos foram analisados quanto à perda de peso e à perda de qualidade (peso hectolítrico e teor de umidade). Para avaliação do peso hectolítrico $(\mathrm{PH})$ utilizou-se uma balança com capacidade para um quarto de litro, da marca Dalemole. A pesagem dos grãos foi feita em uma balança analítica, da marca Gehaka, modelo BG 2000, com precisão de 0,01 g. O teor de umidade dos grãos foi obtido em um determinador do tipo indireto, método de resistência elétrica, modelo universal da marca Gehaka, devidamente aferido com o método padrão de estufa.

O PH e a massa final para cada nível de infestação, foram corrigidos para o teor de umidade inicial dos grãos, $12,5 \%$ b.u. utilizando-se a Eq. 1, citada por Brooker et al. (1992).

$$
\mathrm{U}=\frac{\mathrm{Pa}}{\mathrm{Pa}+\mathrm{Pms}} 100
$$

em que:

$$
\begin{array}{ll}
\mathrm{U} & \text { - teor de umidade, porcentagem base úmida, \% b.u. } \\
\mathrm{Pa} & \text { - massa de água, } \mathrm{kg} \\
\mathrm{Pms} & \text { - massa de matéria seca, } \mathrm{kg}
\end{array}
$$

Para a correção do PH subtraiu-se do peso hectolítrico final (produto infestado e com maior teor de umidade) a quantidade de água responsável por sua redução, em função do aumento do teor de umidade. Desta forma, a causa da diminuição do $\mathrm{PH}$ passa a ser exclusiva da atividade dos insetos. A correção da massa final foi feita de acordo com a descrição a seguir. Após se calcular a massa de água presente na amostra inicial (sem insetos) de $1500 \mathrm{~g}$ e com teor de umidade de $12,5 \%$ b.u., com este mesmo valor se fez a correção para a amostra final (com insetos) geralmente com teor de umidade superior ao inicial; encontrando-se sempre um valor para a massa total final inferior à massa total inicial; assim, para explicitar o resultado da atividade dos insetos na redução da massa, diminuía-se, da massa total final, o valor da massa de água devido ao aumento do teor de umidade.

Utilizando-se os dados do valor de mercado do grão de trigo importado e nacional (cedidos por uma indústria moageira) do custo de controle para fumigação (cedidos por uma empresa do ramo) e do valor obtido pela inclinação das curvas de regressão entre perda de peso $(\mathrm{g})$ e $\mathrm{PH}\left(\mathrm{g} 250 \mathrm{~mL}^{-1}\right)$ em relação à infestação final de insetos, calculou-se o NDE pela Eq. 2, apresentada por Pedigo (1986).

$$
\mathrm{NDE}=\frac{\mathrm{c}}{\mathrm{Vb}}
$$

em que:

c - custo de controle dos insetos, US\$

$\mathrm{V}$ - valor comercial do produto, US\$

b - inclinação da curva, adimensional

O custo de controle dos insetos (c) foi obtido através do valor de mercado cobrado pelas empresas controladoras de pragas para esse tipo de atividade, na qual se inclui o processo de amostragem dos grãos; neste caso, o valor foi de US\$ 0,40 por tonelada. Para o valor comercial do produto, estabeleceramse e se prefixaram os valores antes da variação cambial sofrida no País, no mês de fevereiro de 1999, ou seja, foi de US\$215,00 para o grão importado (Argentino) cedido pela Empresa Moinhos Vera Cruz, safra 1997, e US\$ 118,26 para o grão nacional, oriundo de São Gotardo, MG, safra 1998.

Os dados foram interpretados por meio de análise de regressão, utilizando-se o procedimento PROC REG (SAS, 1987) e análise de regressões não lineares, através do procedimento "curve fitting" do programa Sigma Plot, que descreveu as interações entre os parâmetros avaliados.

\section{RESULTADOS E DISCUSSÃO}

A relação entre as variáveis, peso hectolítrico $\left(\mathrm{g} 250 \mathrm{~mL}^{-1}\right)$, massa final dos grãos (g) e a infestação final dos insetos, foi significativa e linear negativa, ou seja, diminuiu com o aumento da população de $S$. zeamais, em todos os tratamentos, depois de três meses de armazenamento (respectivamente, $\mathrm{R}^{2}=0,92 \mathrm{e}$ $\mathrm{p}<0,0001 ; \mathrm{R}^{2}=0,94$ e $\mathrm{p}<0,0001$ ) (Fig. 1). A redução do $\mathrm{PH}$ chegou a $43 \%$ e a da massa final a $21 \%$, quando a população de insetos inicial foi de 300 indivíduos para $1,5 \mathrm{~kg}$ de grãos, depois de três meses de armazenamento. As análises de perda de peso (g) e do teor de umidade dos grãos $(\%)$ em relação à infestação

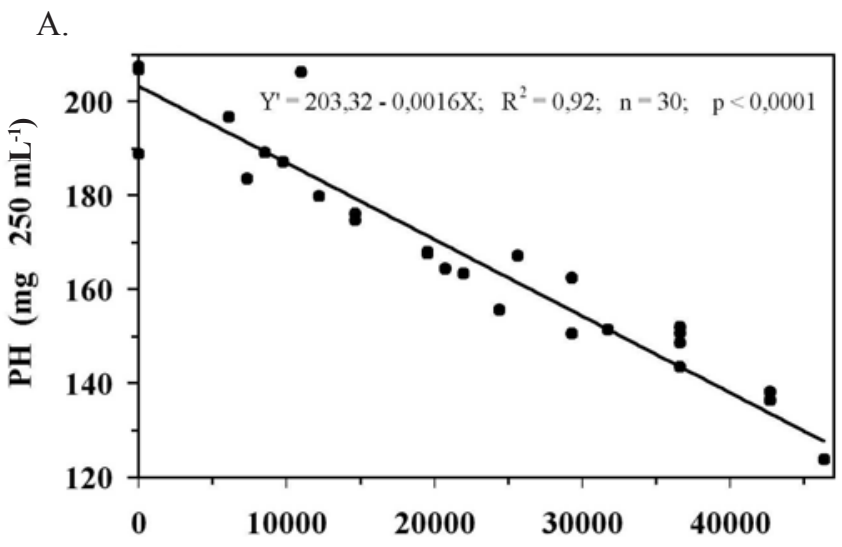

B.

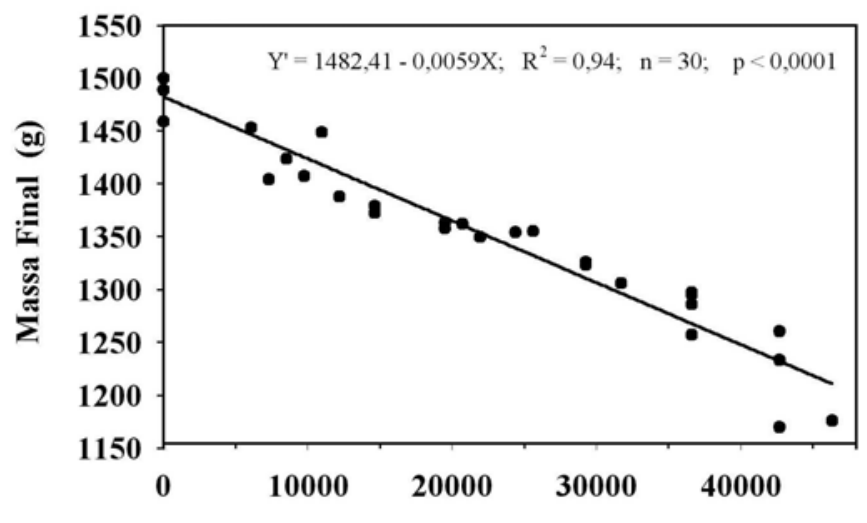

$\mathbf{N}^{\circ}$ Final de Insetos (por 1,5 kg Grão)

Figura 1. Efeito da infestação final de S. zeamais no peso hectolítrico (A) e na massa final (B) dos grãos, após três meses de armazenamento (junho-agosto/98) 
final dos insetos (Fig. 2), para todos os tratamentos, foram significativas, obtendo-se uma relação linear positiva ao fim do período de armazenamento $\left(R^{2}=0,94\right.$ e $\mathrm{p}<0,0001 ; \mathrm{R}^{2}=0,80$ e $\mathrm{p}<0,0001$, respectivamente). Com a redução da massa final de grãos tem-se, conseqüentemente, maior perda de peso (Fig. 2A). Resultados similares foram observados por Anderson et al. (1990) e Reed et al. (1990). Observou-se, também, estreita relação entre o aumento da população de insetos e o teor de umidade dos grãos (Fig. 2B) e acréscimo de até $100 \%$ no teor de umidade dos grãos, quando o número de insetos foi de 300 para cada $1,5 \mathrm{~kg}$ de grãos. De acordo com Flinn \& Hagstrum (1990), Hagstrum \& Flinn (1990) e Hagstrum \& Flinn (1992) o aumento de teor de umidade é ocasionado pelo aumento da taxa respiratória dos insetos.

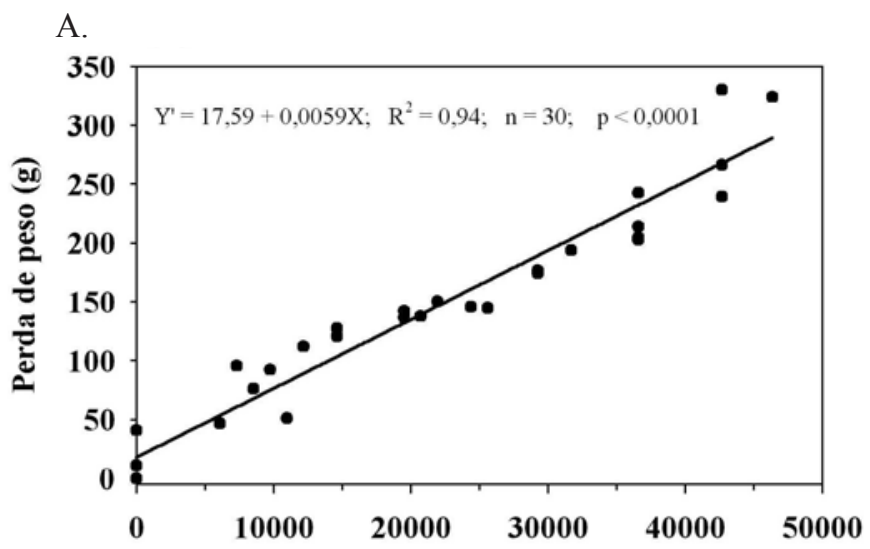

B.

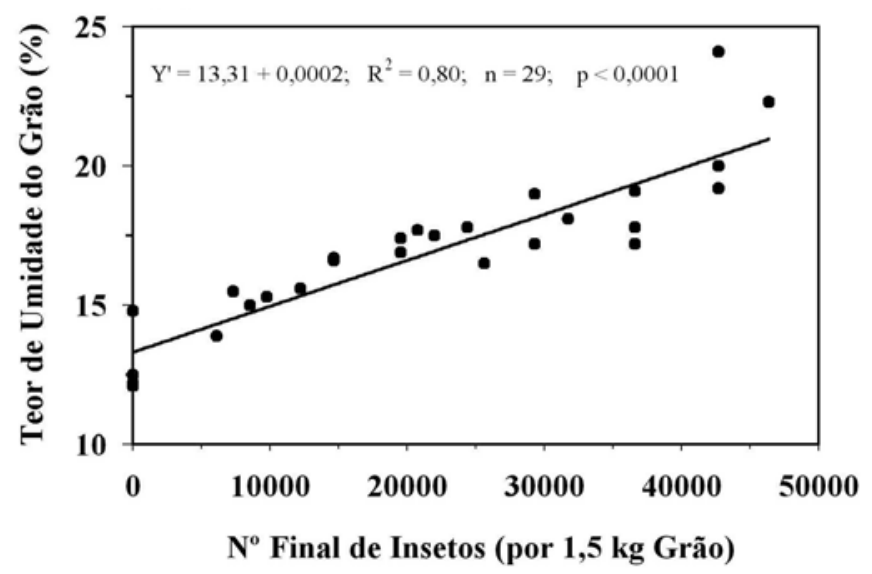

Figura 2. Relação entre a perda de peso (A) e o teor de umidade do grão (B) em relação à infestação final de $S$. zeamais, após três meses de armazenamento (junho-agosto/98)

Quanto ao efeito da densidade populacional dos insetos sobre a temperatura da massa de grãos (Fig. 3), obteve-se uma relação positiva, porém não linear, $\left(\mathrm{R}^{2}=0,5230\right.$ e $\left.\mathrm{p}<0,0017\right)$ significando que, acima de determinada densidade de insetos, a temperatura se manteve em equilíbrio, não interferindo no crescimento populacional da espécie estudada. A partir de uma densidade populacional de 10 indivíduos para cada $1,5 \mathrm{~kg}$ de grãos de trigo, a temperatura permanece praticamente constante. Segundo Salom et al. (1987) a temperatura é uma variável que pode determinar a taxa de crescimento, desenvolvimento e reprodução dos insetos. Temperaturas acima de $5^{\circ} \mathrm{C}$ da temperatura ótima de desenvolvimento dos insetos, podem causar a morte, retardar ou paralisar os processos fisiológicos e comportamentais e, em alguns casos, reduzir a natalidade e modificar o padrão de dispersão (Hagstrum et al., 1996).

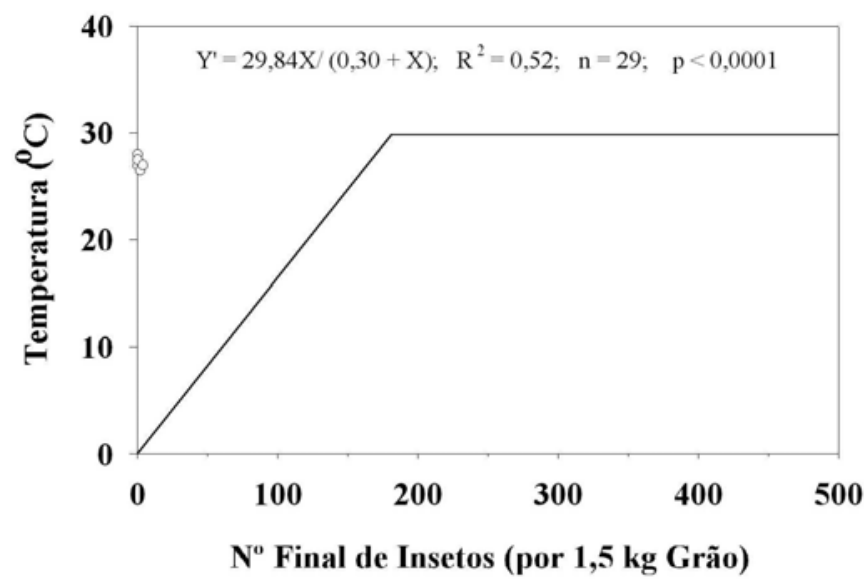

Figura 3. Efeito da infestação final de $S$. zeamais na temperatura da massa de grãos, após três meses de armazenamento (junhoagosto/98)

As mesmas tendências foram obtidas quando se avaliaram os valores iniciais da população de insetos em relação às variáveis em estudo. Os resultados foram lineares para o $\mathrm{PH}$ (g $250 \mathrm{~mL}^{-1}$ ) e massa final da amostra (g) - Figura 4, perda de peso
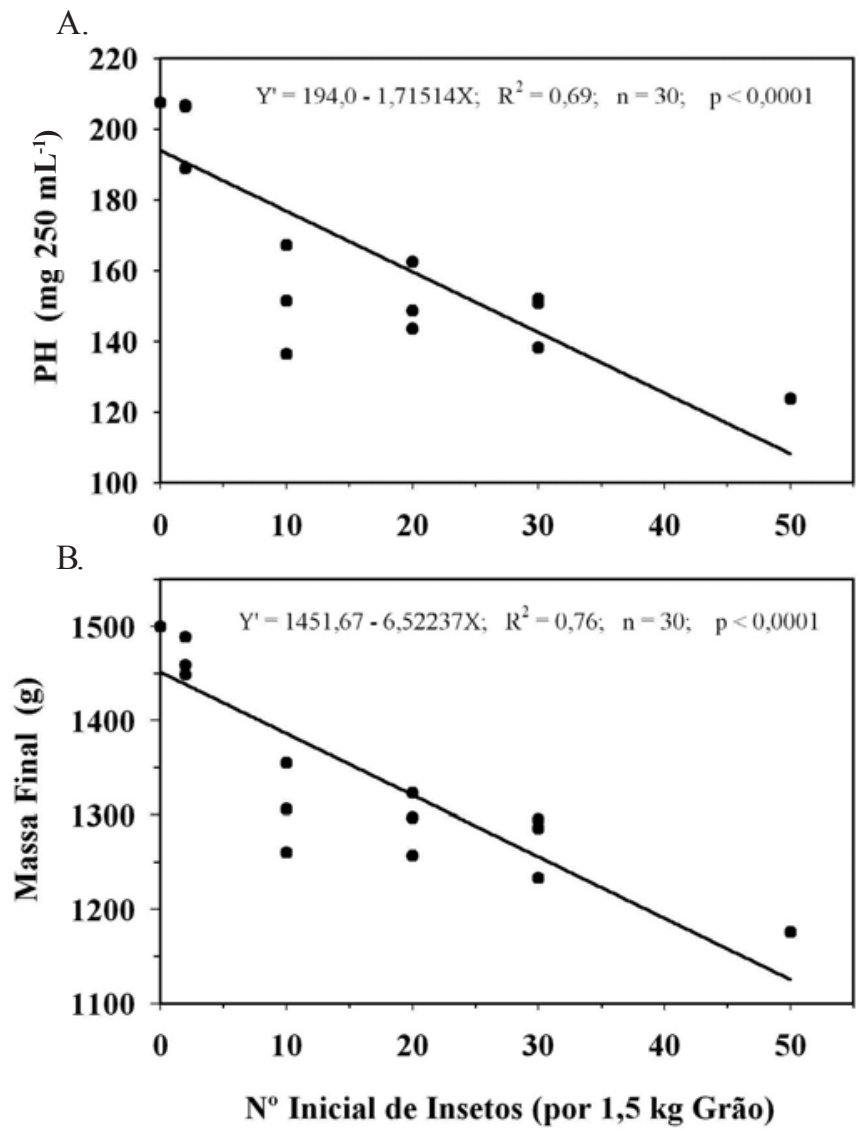

Figura 4. Efeito da infestação inicial de $S$. zeamais no peso hectolítrico (A) e na massa final (B) dos grãos, após três meses de armazenamento (junho-agosto 98) 
(g) e teor de umidade dos grãos (\%) - Figura 5; para o PH e a massa final, encontrou-se uma correlação significativa e linear negativa $\left(R^{2}=0,69\right.$ e $p<0,0001 ; R^{2}=0,76$ e $p<0,0001$, respectivamente) e, para a perda de peso e umidade dos grãos, uma relação significativa linear, porém positiva $\left(\mathrm{R}^{2}=0,76 \mathrm{e} \mathrm{p}<\right.$ 0,$0001 ; R^{2}=0,71$ e p $<0,0001$, respectivamente). Para o efeito da temperatura $\left(R^{2}=0,54\right.$ e p $\left.=0,0017\right)$ - Figura 6 , encontrou-se o mesmo comportamento observado na Figura 3, em que, a partir de 10 indivíduos para cada $1,5 \mathrm{~kg}$ de grãos, a temperatura não atinge patamares muito superiores aos dos tratamentos com menor número de insetos e para o mesmo período de armazenamento. A finalidade de se obter essas relações é, por exemplo, se conhecer o número de insetos em determinada massa de grãos, pelo processo de amostragem, e poder inferir suas conseqüências depois de três meses de armazenamento, quando não é aplicada nenhuma tática de controle.
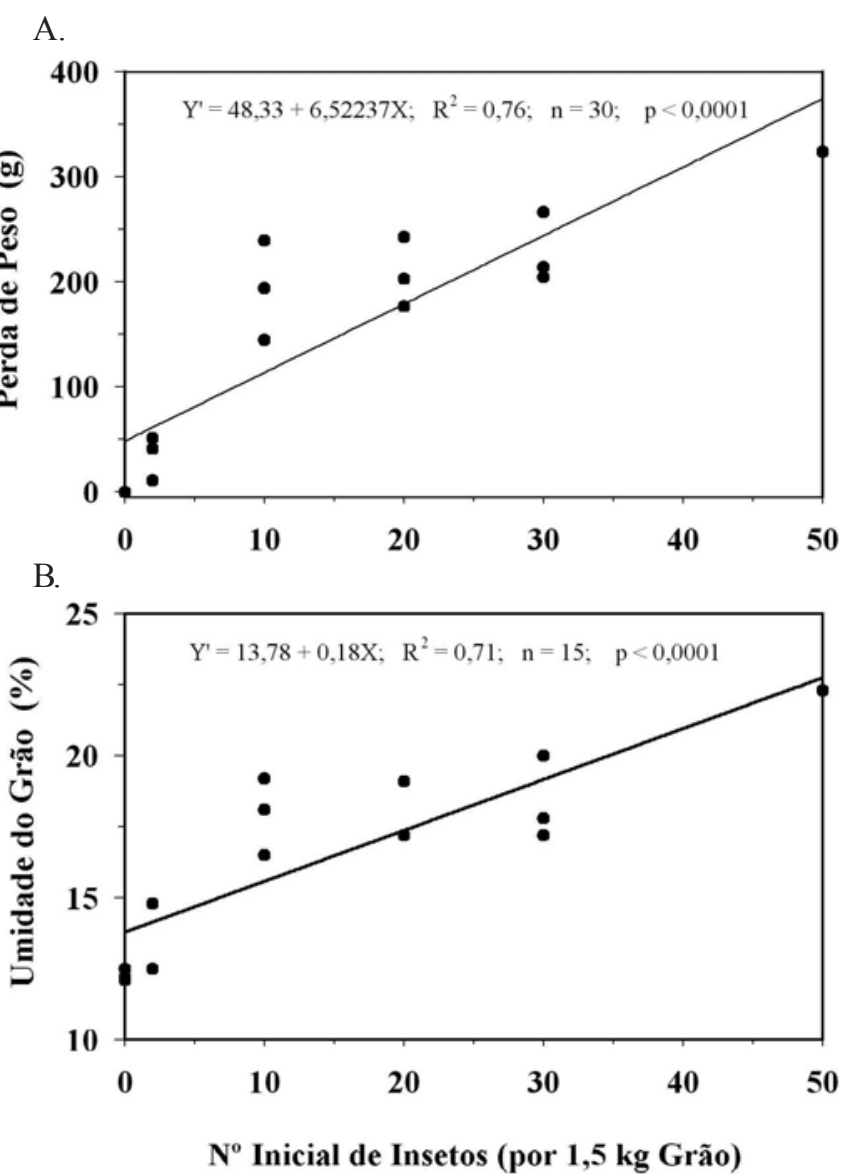

Figura 5. Efeito da infestação inicial de $S$. zeamais na perda de peso (A) e no teor de umidade dos grãos (B) após três meses de armazenamento (junho-agosto/98)

A relação entre as infestações iniciais e finais, foi positiva (Fig. 7), demonstrando que a densidade populacional aumenta à medida que aumenta o número de insetos $\left(\mathrm{R}^{2}=0,88 \mathrm{e} \mathrm{p}<\right.$ $0,0001)$. Ressalta-se, no entanto, que esta relação só foi possível até a densidade de 50 insetos para $1,5 \mathrm{~kg}$ de grãos de trigo pois, a partir desta população, ocorreu grande flutuação populacional, não se encontrando uma equação matemática que se ajustasse aos dados. Segundo Sauer et al. (1992) uma possível explicação para flutuação populacional, é que a

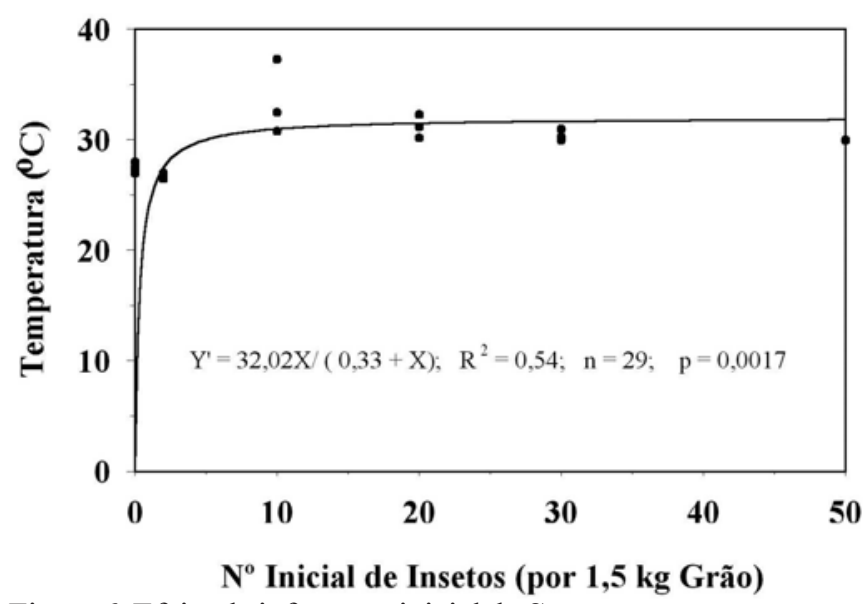

Figura 6. Efeito da infestação inicial de $S$. zeamais na temperatura da massa, ao fim dos três meses de armazenamento dos grãos de trigo (junho-agosto/98)

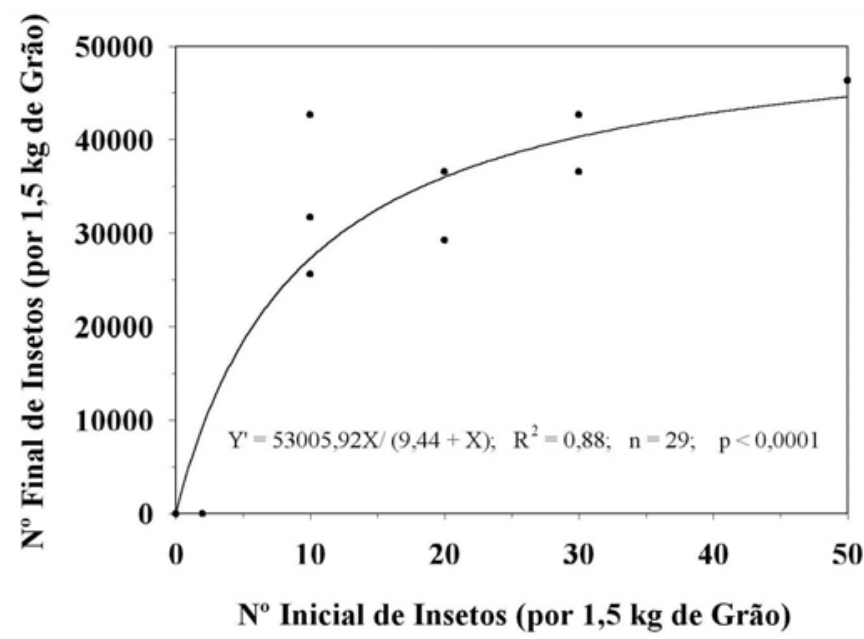

Figura 7. Relação entre o número inicial de $S$. zeamais inseridos em 1,5 kg de grãos de trigo e a infestação final obtida após três meses de armazenamento (junho-agosto/98)

elevação do teor de umidade dos grãos (Fig. 2B), favorece o desenvolvimento de microrganismos e, conseqüentemente, afeta o crescimento populacional dos insetos.

A relação encontrada entre o valor comercial (US\$) por tonelada de grão, importado e nacional, e o número inicial de insetos por 1,5 kg grãos (Figs. 8A e B), foi linearmente negativa, demonstrando a depreciação desta matéria-prima a medida em que se aumenta o número de insetos após três meses de armazenamento (respectivamente, $R^{2}=0,76 \mathrm{e} p<0,0001 ; \mathrm{R}^{2}=0,76 \mathrm{e}$ $\mathrm{p}<0,0001)$. A mesma relação foi observada entre o número de insetos finais, inseridos em $1,5 \mathrm{~kg}$ de grãos, e o valor comercial dos grãos, importado e nacional - Figuras $8 \mathrm{C}$ e $\mathrm{D}$ (respectivamente, $\mathrm{R}^{2}=0,94$ e $\mathrm{p}<0,0001 ; \mathrm{R}^{2}=0,94$ e $\mathrm{p}<0,0001$ ).

Com base na redução do peso da massa de grãos e PH e no custo de controle para trigo utilizando-se o tratamento de fumigação dos grãos com fosfina, calculou-se o nível de dano (NDE) para o $S$. zeamais; para trigo nacional, o NDE foi de 1,37 insetos $\mathrm{kg}^{-1}$ para a relação com $\mathrm{PH}$ e de 0,33 insetos $\mathrm{kg}^{-1}$ quanto à perda de peso. Para o trigo importado, o NDE foi de 0,76 insetos $\mathrm{kg}^{-1}$ para a relação com $\mathrm{PH}$ e de 0,18 insetos $\mathrm{kg}^{-1} \mathrm{em}$ relação à perda de peso, depois de três meses de armazenamento. 


\section{A.}

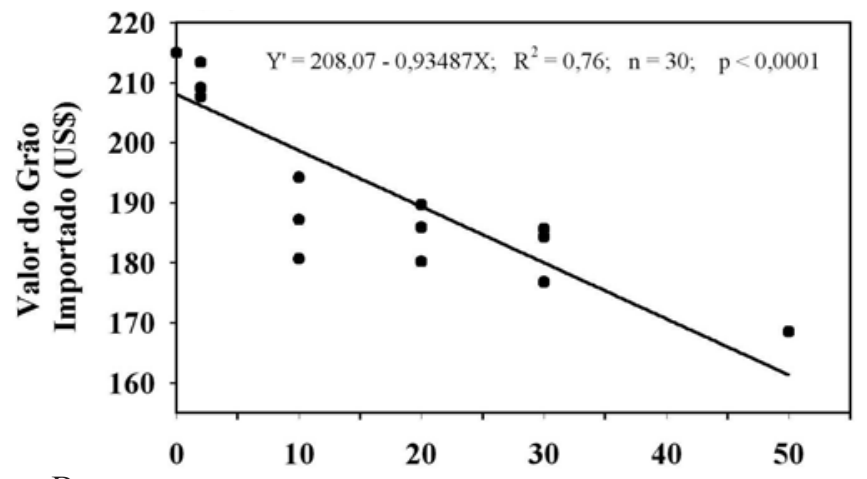

B.

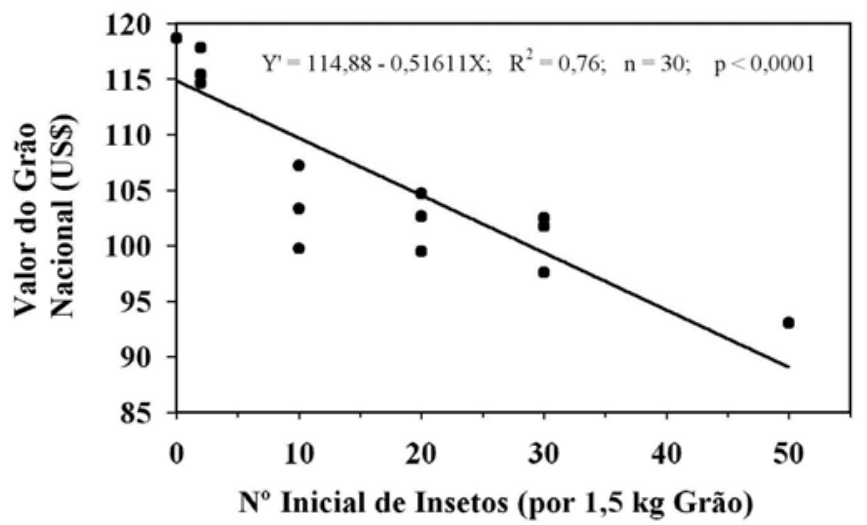

C.
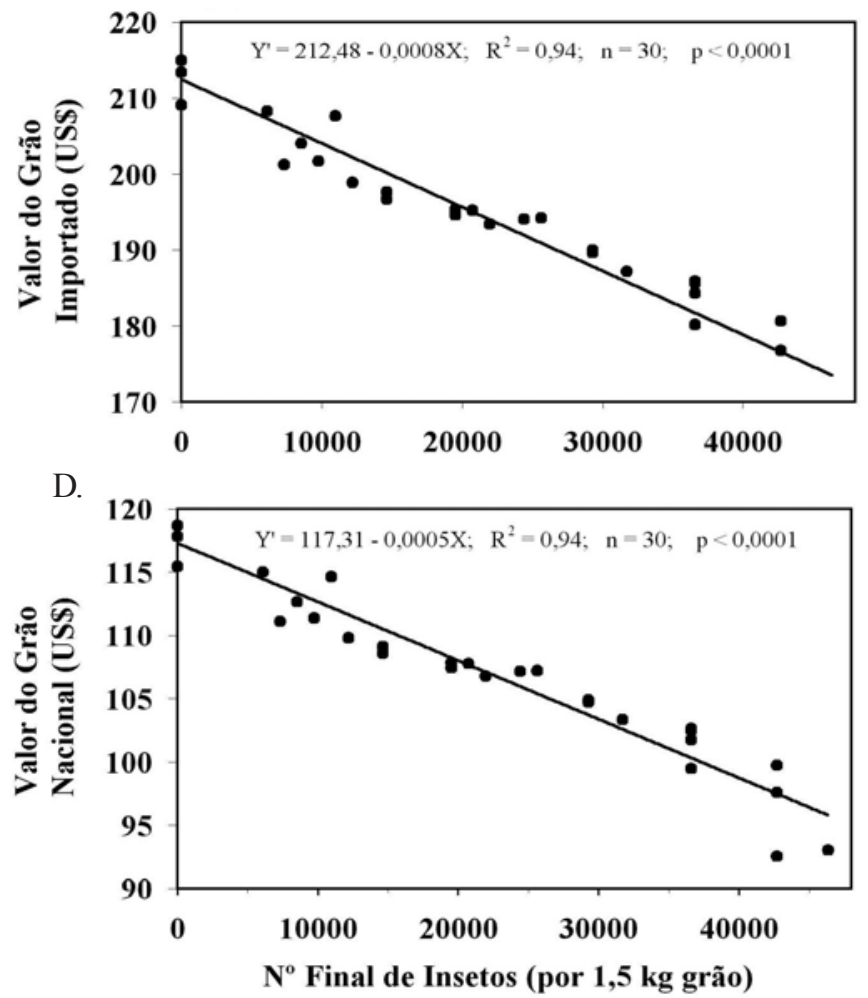

Figura 8. Desvalorização comercial do grão importado (A e C) e nacional (B e D) em dólar, em relação à infestação inicial e final de $S$. zeamais após três meses de armazenamento

Ante esses aspectos, calculou-se o nível de dano econômico (NDE) que associa a qualidade do grão e seu rendimento em farinha de trigo, em relação à presença de insetos na massa de grãos. Este índice permite estabelecer-se limites quanto à densidade populacional (número de indivíduos por kg de trigo) no processo de amostragem, durante o recebimento e armazenamento dos grãos, relacionando-o ao tempo previsto de armazenamento (Hagstrum et al., 1996).

O NDE obtido $\left(0,18\right.$ inseto $\left.\mathrm{kg}^{-1}\right)$ é muito baixo e sugere que, em um processo de recebimento da matéria-prima, bem como na amostragem durante o armazenamento, qualquer inseto vivo encontrado na amostra $(\mathrm{kg})$ da massa levará a uma desvalorização qualitativa e quantitativa dos grãos e, ainda, que uma ação de controle deverá ser aplicada de imediato. Este resultado sugere um controle da massa de grãos logo após a recepção, caso não haja um programa de monitoramento e tomada de decisões. Segundo Subramanyam \& Hagstrum (1996) o custo de controle é menor que o de amostragem e o tempo gasto para realizá-la. Hagstrum \& Flinn (1996) sugerem, para condições temperadas, que as técnicas de controle para insetos de grãos armazenados sejam aplicadas quando a população de insetos atingir a faixa de 0,1 a 5,0 indivíduos por kg de grãos.

Para indústrias de alimento, a perda de qualidade devido à infestação por insetos é mais preocupante que a perda de peso, mas a quantificação da perda de qualidade e sua associação a um valor monetário, é mais difícil que os cálculos para a perda de peso (Olkowski, 1974; Zungoli \& Robinson, 1984; Raupp et al., 1987).

A redução do valor comercial, tanto para o grão importado quanto para o nacional, está diretamente relacionada ao número de insetos presentes na massa de grãos (Fig. 8). A medida em que se aumenta o número de insetos, maior também é a depreciação da matéria-prima durante o armazenamento, pois há redução da massa final de grãos e no peso hectolítrico do grão, o que influenciará no rendimento de farinha durante a moagem. O valor do NDE calculado é momentâneo, pois ele depende do valor comercial do produto, que varia entre safras e depende da demanda do mercado.

A análise de custo-benefício fornece, ao programa de manejo integrado, uma ferramenta para a implementação de táticas de controle mais corretas e em momentos propícios para o controle das pragas (Sauer et al., 1992; Hagstrum \& Flinn, 1996); no entanto, de acordo com Hagstrum \& Flinn (1996) uma medida de controle é economicamente eficiente se o valor do grão, no momento da venda menos o custo de amostragem e controle for maior que o valor do grão sem controle; neste caso, observou-se, no presente trabalho que, face ao baixo NDE para S. zeamais em armazenamento de trigo durante o período de três meses sem medidas de controle, é freqüentemente justificável o pronto tratamento de trigo no ato de sua recepção na unidade armazenadora.

\section{CONCLUSÕES}

Após três meses de armazenamento, concluiu-se que:

1. Os valores do nível de dano econômico foram de 1,37 insetos $\mathrm{kg}^{-1}$ em função do peso hectolítrico e de 0,33 inseto $\mathrm{kg}^{-1}$ em relação à perda de peso para o trigo nacional.

2. Para o trigo importado, os valores do nível de dano econômico foram de 0,76 insetos $\mathrm{kg}^{-1}$ para a relação com o peso hectolítrico e de 0,18 insetos $\mathrm{kg}^{-1} \mathrm{em}$ relação à perda de peso. 


\section{LITERATURA CITADA}

Anderson, K.; Schurle, B.; Reed, C.; Pedersen, J. An economic analysis of producers decisions regarding insect control in stored grain. North Central Journal of Agricultural Economics, Urbana, v.12, p. 23-29, 1990.

Brasil. Ministério da Agricultura e do Abastecimento. Secretaria de Desenvolvimento Rural. Norma de identidade e qualidade do trigo. Brasília, 1998. 3p. Portaria 268, 21/09/1998

Brooker, D.B.; Bakker-Arkema, F.W.; Hall, C.W. Drying and storage of grain and oilseeds. Westport: AVI; New York: van Nostrand Reinhold, 1992.450p.

Brower, J.H.; Smith, L.; Vail, P.V.; Flinn, P.W. Biological control. In: Subramanyam, B.; Hagstrum, D.W. (eds.) Integrated management of insects in stored products. New York: M. Dekker, 1996. p.223-286.

Chant, D.A. Integrated control systems. In: Scientific aspects of pest control. Symposium arranged and conducted by the National Academy of Sciences, National Research Council, at Washington, D.C., 1966. Proceedings... Washington, 1966. p.193-198. National Research Council (US). Publication, 1402

Flinn, P.W.; Hagstrum, D.W. Simulations comparing the effectiveness of various stored-grain management practices used to control the lesser grain borer, Rhyzopertha dominica (F.) (Coleoptera: Bostrichidae). Environmental Entomology, College Park, v.19, n.3, p.725-729, 1990.

Hagstrum, D.W.; Flinn, P.W. Simulations comparing insect species differences in response to wheat storage conditions and management practice. Journal of Economic Entomology, Lanham, v.83, n. 6, p.2469-2475, 1990.

Hagstrum, D.W.; Flinn, P.W. Integrated pest management of stored-grain insects. In: Sauer, D.B. (eds.). Storage of cereal grains and their products. 4.ed. St. Paul: American Association of Cereal Chemists, 1992. p.535-562.

Hagstrum, D.W.; Flinn, P.W. Integrated pest management. In: Subramanyam, B.; Hagstrum, D.W. (eds.) Integrated management of insects in stored products. New York: M. Dekker, 1996. p.399-409.

Hagstrum, D.W.; Flinn, P.W.; Howard, R.W. Ecology. In: Subramanyam, B.; Hagstrum, D.W. (eds.) Integrated management of insects in stored products. New York: M. Dekker, 1996. p.71-134.

Harein, P.K.; Davis, R. Control of stored-grain insects. In: Sauer, D.B. (ed.). Storage of cereal grains and their products. 4.ed. St. Paul: American Association of Cereal Chemists, 1992. p.491-534.

Mills, R.; Pedersen, J.R. A flour mill sanitation manual. St. Paul: Eagan Press, 1990. 64p.

Okelana, F.A.; Osuji, F.N.C. Influence of relative humidity at $30{ }^{\circ} \mathrm{C}$ on the oviposition, development and mortality of Sitophilus zeamais Motschulsky (Coleoptera:Curculionidae) in maize kernels. Journal of Stored Products Research, Oxford, v.21, n.1, p.13-19, 1985.

Olkowski, W. A model ecosystem management program. In: Tall Timbers Conference on Ecological Animal Control by Habitat Management, 1964, Talahassee. Proceedings... Talahassee: Tall Timbers Research Station, 1974. v.5, p.103-117.
Onstad, D.W. Calculation of economic-injury levels and economics thresholds for pest management. Journal of Economic Entomology, Lanham, v.80, n.2, p.297-303, 1987.

Pedersen, J.R. Insects: identification, damage and detection. In: Sauer, D.B. (ed.). Storage of cereal grains and their products. 4.ed. St. Paul: American Association of Cereal Chemists, 1992. p.435-489.

Pedigo, L.P.; Hutchins, S.H.; Higley, L.G. Economic injury levels in theory and practice. Annual Review of Entomology, Palo Alto, v.31, p.341-68, 1986.

Raupp, M.J.; Davidson, J.A.; Koehler, C.S.; Sadof, C.S.; Reichelderfer, K. Decision-making considerations for aesthetic damage caused by pests. Bulletin of the Entomological Society of America, College Park, v.34, n.1, p.27-32, 1987.

Reed, C.; Anderson, K.; Brockschmidt, J.; Wright, V.; Pedersen, J.R. Cost and effectiveness of chemical insect control measures in farm-stored Kansas wheat. Journal of the Kansas Entomological Society, Manhattan, v.63, n.3, p.351-360, 1990.

Rees, D.P. Coleoptera. In: Subramanyam, B.; Hagstrum, D.W. (eds.) Integrated management of insects in stored products. New York: M. Dekker, 1996. p.1-39.

Salom, S.M.; Stephen, F.M.; Thompson, L.C. Development rates and a temperature-dependent model of pales weevil, Hylobius pales (Herbst), development. Environmental Entomology, College Park, v.16, n.4, p.956-962, 1987.

Sauer, D.B.; Meronuck, R.A.; Christensen, C.M. Microflora. In: Sauer, D.B. (ed.). Storage of cereal grains and their products. 4.ed. St. Paul: American Association of Cereal Chemists, 1992. p.313-340.

Schöller, M.; Prosell, S.; Al-Kirshi, A.G.; Reichmuth, C.H. Towards biological control as a major component of integrated pest management in stored product protection. Journal of Stored Products Research, Oxford, v.33, n.1, p.81-97, 1997.

Sinha, R.N. The stored-grain ecosystem. In: Jayas, D.S.; White, N.D.G.; Muir, W.E. (eds.). Stored-grain ecosystems. New York: M. Dekker, 1995, p.1-33.

Stern, V.M. Significance of the economic threshold in integrated pest control; summary of session and discussion. In: FAO Symposium on Integrated Pest Control, 1965, Rome. Proceedings... Rome: FAO, 1966. v.2, p.41-56.

Stern, V.M.; Smith, R.F.; van den Bosh , R.; Hagen, K.S. The integrated control concept. Hilgardia, Berkeley, v.29, n.2, p.81-101, 1959.

Subramanyam, B.; Hagstrum, D.W. Sampling. In: Subramanyam, B.; Hagstrum, D.W. (eds.) Integrated management of insects in stored products. New York: M. Dekker, 1996. p.135-193.

White, N.D.G.; Leesch, J.G. Chemical control. In: Subramanyam, B.; Hagstrum, D.W. (eds.) Integrated management of insects in stored products. New York: M. Dekker, 1996. p.287-330.

Zungoli, P.A.; Robinson, W.H. Feasibility of establishing an aesthetic injury level for German cockroach pest management programs. Environmental Entomology, College Park, v.13, n. 6, p.1453-1458, 1984. 